\title{
Downregulation of pancreatic-duodenal homeobox 1 expression in breast cancer patients: A mechanism of proliferation and apoptosis in cancer
}

\author{
JIE MA, JIA LI, HUA LI, XIGANG XIAO, LEI SHEN and LIN FANG
}

Department of Breast and Thyroid Surgery, Tenth People's Hospital of Tongji University, Zhabei, Shanghai 200072, P.R. China

Received March 28, 2012; Accepted August 7, 2012

DOI: $10.3892 / \mathrm{mmr} .2012 .1067$

\begin{abstract}
Pancreatic-duodenal homeobox 1 (PDX-1) is a transcription factor that regulates embryological pancreas development and insulin expression in adult islets. The current study investigated the expression profile and potential role of PDX-1 in breast cancer. Immunohistochemistry was performed to determine the expression pattern of PDX-1 in breast cancer and adjacent benign breast tissues. In addition, cell proliferation and the cell cycle were evaluated following the transient inhibition of PDX-1 with antisense oligonucleotides in MCF-7 human breast cancer cells. Real-time PCR and western blotting were conducted to investigate the correlation between PDX-1, P53, Ki-67, Caspase 3 and Caspase 8. These experiments demonstrated that PDX-1 was downregulated in human breast cancer tissue compared with adjacent normal breast tissue. Knockdown of PDX-1 expression in vitro in MCF-7 breast cancer cells promoted cell proliferation and disrupted the cell cycle, as demonstrated by the overexpression of P53 and $\mathrm{Ki}-67$ at the mRNA and protein levels. In conclusion, the current study shows that PDX-1 regulates cell proliferation and the cell cycle in human breast cancer cells by altering the expression of the cell cycle-related genes, P53 and Ki-67. These data suggest that PDX-1 is a putative tumor suppressor in breast cancer.
\end{abstract}

\section{Introduction}

The pancreatic-duodenal homeobox protein 1 (PDX-1) is $6 \mathrm{~kb}$ in length and located on chromosome 5 in mice, 12 in rats and 13q12.1 in humans (1). During mouse embryogenesis, PDX-1 is expressed in the pancreas, duodenum and gastric pyloric glands. These organs are deformed in PDX1-deficient mice (2). As a transcription factor, the PDX-1 gene plays a critical role in embryological pancreas development and insulin expres-

Correspondence to: Professor Lin Fang, Department of Breast and Thyroid Surgery, Tenth People's Hospital of Tongji Univeristy, 301 Yanchang Road, Zhabei, Shanghai 200072, P.R. China

E-mail: fanglin_f@126.com

Key words: breast cancer, PDX-1, tumur suppressor gene sion in adult pancreatic islets $(3,4)$. PDX-1 deficiency leads to duodenal and pancreatic ageing (5). The name PDX-1 is inaccurate, as the transcription factor is not exclusively expressed in pancreatic and duodenal tissues and is highly expressed by a large proportion of human tumors, including gastric and pancreatic cancer.

Over the past decade, there has been a large body of evidence supporting the aberrant expression of PDX-1 in malignant tumors, including pancreatic $(6)$, prostate $(7)$, stomach $(8,9)$, colon $(10)$ and breast cancers $(11,12)$. In pancreatic cancer, PDX-1 induces increased cell proliferation, invasion and colony formation in vitro. PDX-1 expression has also been correlated with human embryonic kidney (HEK) 293 and MIA PaCa2 pancreatic cancer cell xenograft tumor formation in SCID mice. Thus, these data suggest that PDX-1 has oncogenic properties (13). Another study reported that the expression level of PDX-1 correlates with lymph node metastasis and histological grade, where positive PDX-1 was associated with poor prognosis for breast cancer patients $(14,15)$, while Ma et al demonstrated a tumor suppressor role of PDX-1 in gastric cancer (16).

However, at present, little is known about the expression and function of PDX-1 in breast cancer. In this study, the expression of PDX-1 in breast cancer specimens and adjacent normal tissues was examined using immunohistochemistry, and staining was scored against pathological characteristics. Antisense oligonucleotide (ASO) was employed to downregulate $\mathrm{PDX}-1$ expression in $\mathrm{MCF}-7$ breast cancer cells and to determine its impact on cell proliferation and the cell cycle.

\section{Materials and methods}

Specimens. In this study, 81 formalin-fixed paraffin-embedded human breast cancer specimens and 20 adjacent normal specimens were collected from the Department of Pathology of Shanghai Tenth People's Hospital. All samples were confirmed as invasive ductal breast cancer. The specimens were from patients who had not received any chemotherapy or radiotherapy prior to surgery.

Immunohistochemistry. Paraffin-embedded breast cancer sections were heated to $75^{\circ} \mathrm{C}$ for $60 \mathrm{~min}$, deparaffinized by dimethyl benzene (Guoyao Corp., Shanghai, China), treated with $3.0 \% \mathrm{H}_{2} \mathrm{O}_{2}$ in methanol for $10 \mathrm{~min}$ and rehydrated in 
graded concentrations of ethanol/water. Antigen retrieval was accomplished by heating in $10 \mathrm{mmol}$ citrate buffer, $\mathrm{pH}$ 6.0, for $30 \mathrm{~min}$ in a steamer, followed by cooling for $30 \mathrm{~min}$ at room temperature. Sections were blocked with 5\% BSA for 30 min then incubated with primary antibody against PDX-1, $\mathrm{Ki}-67$ or P53 for $2 \mathrm{~h}$ at $37^{\circ} \mathrm{C}$. Indirect immunohistochemical detection was performed through incubation with 200 -fold diluted peroxidase-conjugated secondary antibodies (Santa Cruz Biotechnology, Inc., Santa Cruz, CA, USA) for $60 \mathrm{~min}$ at room temperature. The peroxidase reaction was developed using diaminobenzidine (Sigma-Aldrich, Shanghai, China), followed by counterstaining with Harris' hematoxylin, dehydration and mounting.

PDX-1 staining for each tissue core was semi-quantitatively scored as 0-1 (-), $2(+), 3-4(++)$ and 5-6 (+++), according to the amount of stained cells and the intensity of staining. High-power magnification fields (five) were randomly selected: (a) Intensity of staining: no brown-yellow, 0 ; slightly yellow, 1; brown-yellow, 2; brown, 3; (b) The percentage of stained cells: no PDX-1-stained cells or PDX-1 stained cells account for less than $5 \%, 0 ; \leq 25 \%, 1 ; 26-50 \%, 2$; (c) The final score was determined by the sum of (a) and (b).

Cell culture. The MCF-7 breast cancer cell line used in this study was obtained from the Chinese Academy of Sciences and cultured in DMEM (Gibco, New York, NY, USA) supplemented with $10 \%$ fetal bovine serum (Gibco), $100 \mathrm{U} / \mathrm{ml}$ penicillin and $100 \mu \mathrm{g} / \mathrm{ml}$ streptomycin (Genom, Hangzhou, China). Cells were incubated at $37^{\circ} \mathrm{C}$ in a humidified chamber supplemented with $5 \% \mathrm{CO}_{2}$.

ASO transfection. For transfections, $2-4 \times 10^{6}$ cells/well were seeded into a 6-well plate and cultured in serum- and antibiotic-free DMEM. As the confluency of MCF-7 breast cancer cells reached $80-90 \%$, cells were transfected with ASO. Briefly, ASO plasmid and lipofect transfection reagent were independently diluted in $250 \mu \mathrm{l}$ DMEM medium at a ratio of 1:3 ASO:lipofect and incubated for $5 \mathrm{~min}$ at room temperature. Next, the diluted ASO plasmid and lipofect were gently combined and incubated for a further $20 \mathrm{~min}$. Following this, $500 \mu \mathrm{l}$ of the ASO-lipofect complex was added to each well. DMEM was replaced by DMEM with 10\% FBS 4-5 h later. All cells were incubated in a $37^{\circ} \mathrm{C} / 5 \% \mathrm{CO}_{2}$ incubator for $48 \mathrm{~h}$ prior to further testing.

Cell proliferation assay. Cell proliferation was assessed using the CCK-8 assay kit (Sigma, Santa Clara, CA, USA). Cells were plated in 96-well plates at 10,000 cells/well (Corning, Inc., Corning, NY, USA). Cells were transfected in triplicate with $0.1,0.2,0.4,0.6$ or $0.8 \mu \mathrm{mol} / 1 \mathrm{PDX}-1$ ASO. Cell proliferation was evaluated at 24,48 and $72 \mathrm{~h}$, according to the manufacturer's instructions.

Cell cycle assay. Cells were harvested and centrifuged at 1,200 rpm for $5 \mathrm{~min}$ and washed twice in PBS. To fix cells, $3 \mathrm{ml}$ ice-cold $70 \%$ ethanol was added in a dropwise fashion and incubated with cells for at least $30 \mathrm{~min}$. A total of $250 \mu \mathrm{l} 0.05 \mathrm{~g} / 1$ propidium iodide (PI) staining solution was added to each sample and incubated for $30 \mathrm{~min}$ at room temperature. PI staining was then analyzed by flow cytometry
(FACSCanto $^{\text {TM }}$ II, BD Biosciences, Piscataway, NJ, USA). The proliferation index was calculated according to the following equation: $\mathrm{PI}=(\mathrm{S}+\mathrm{G} 2 / \mathrm{M}) /(\mathrm{G} 0 / \mathrm{G} 1+\mathrm{S}+\mathrm{G} 2 / \mathrm{M})$.

Real-time PCR analyses. Total RNA was isolated using TRIzol (Invitrogen, Carlsbad, CA, USA) and cDNA was generated by reverse transcription according to the Prime Script TM RT-PCR kit instructions (Takara, Shiga, Japan). Quantitative real-time PCR was performed on a 7900 HT fast RT-PCR Instrument (Applied Biosystems, Singapore) using SYBR-Green as the detection fluorophore. Forward (F) and reverse (R) primers used were as follows: PDX-1, F: 5'-TTAGT GATACTGGATTGGCGTTGT-3'， R: 3'-TGGCTTTAT GGCAGATTAAGGTAC-5'; P53, F: 5'-CCACCATCCACT ACAACTACAT-3', R: 3'-AGGACAGGCACAAACACG-5'; Ki-67, F: 5'-GGGTTACCTGGTCTTAGTT-3', R: 3'-ATG GTTGAGGCTGTTCC-5'; Caspase 3: F: 5'-ATGGAAGCGA ATCAATGGACTC-3', R:3'-CTGTACCAGACCGAGA TGTCA-5'; Caspase 8, F: 5'-CCTGTCACTGTCTTGTA CCCT-3', R:3'-CCCGCAGTATCTTGCCTCC-5' and $\beta$-actin, F: 3'-CTGGAACGGTGAAGGTGACA-5' R: 5'-AGGGTA CATGGTGGTGCCGCCAGAC-3'. PCR parameters were as follows: $2 \mathrm{~min}$ at $95^{\circ} \mathrm{C}$ and then 40 cycles of $15 \mathrm{sec}$ at $95^{\circ} \mathrm{C}$, $30 \mathrm{sec}$ at $60^{\circ} \mathrm{C}$. Each sample was tested in triplicate. Genespecific mRNA expression was normalized against the expression of the internal control, $\beta$-actin.

Western blotting. Protein samples were separated on $12 \%$ SDS-polyacrylamide gel (SDS-PAGE) and transferred onto PVDF membranes (Beyotime, Jiangsu, China). Immune complexes were formed by incubation of the proteins with primary antibodies against PDX-1 (R\&D systems, Minneapolis, MN, USA), P53, Ki-67, Caspase 3 or Caspase 8 (Santa Cruz Biotechnology) overnight at $4^{\circ} \mathrm{C}$. Blots were washed and incubated for $1 \mathrm{~h}$ with DyLight-tagged antibodies conjugated anti-mouse secondary antibodies (R\&D Systems). Immunoreactive protein bands were detected with an Odyssey Scanning System (Li-Cor, Lincoln, NE, USA).

Statistical analysis. All data are representative of at least 3 separate experiments performed in triplicate and are expressed as mean \pm standard deviation. Correlations with tumor characteristics were calculated using Spearman's analysis. As all the data were ranked, the differences were analyzed using a non-parametric two independent samples test (Mann-Whitney U) or $\mathrm{K}$ independent sample test (Kruskal-Wallis Test). The null hypothesis was rejected at the 0.05 level. For RT-PCR analyses, all the samples were tested in triplicate, based on the $2-\Delta \Delta \mathrm{Ct}$ method described by Livac et al (17), and semi-quantitative analysis was used for gene quantification. Paired t-tests were performed to examine the differences between transfection groups and controls in MCF-7 breast cancer cells.

\section{Results}

PDX-1 is underexpressed in breast cancer specimens. To investigate the correlation between PDX-1 and breast cancer, the expression of PDX-1 in human breast cancer specimens and adjacent normal tissues was examined by HE and immunohistochemical staining. Quantitative analysis revealed that 
Table I. PDX-1 expression in breast cancer specimens and adjacent normal breast tissues.

\begin{tabular}{|c|c|c|c|c|c|}
\hline \multirow[b]{2}{*}{ Pathological type } & \multirow[b]{2}{*}{ (n) } & \multicolumn{4}{|c|}{ PDX-1 positive } \\
\hline & & $\begin{array}{c}\text { Negative } \\
(-)\end{array}$ & $\begin{array}{l}\text { Mildly } \\
\text { positive } \\
(+)\end{array}$ & $\begin{array}{c}\text { Moderately } \\
\text { positive } \\
(++)\end{array}$ & $\begin{array}{c}\text { Strongly } \\
\text { positive } \\
(+++)\end{array}$ \\
\hline Breast cancer tissues & 81 & 23 & 19 & 26 & 13 \\
\hline Adjacent normal breast tissues & 20 & 0 & 2 & 7 & 11 \\
\hline
\end{tabular}

PDX-1, pancreatic-duodenal homeobox 1 .

Table II. Clinical characteristics and PDX-1 protein expression in breast cancer.

\begin{tabular}{|c|c|c|c|c|c|c|}
\hline \multirow[b]{2}{*}{ Variable } & \multirow{2}{*}{$\begin{array}{l}\text { No. } \\
\text { (n) }\end{array}$} & \multicolumn{4}{|c|}{ PDX-1 } & \multirow[b]{2}{*}{ P-value } \\
\hline & & - & + & ++ & +++ & \\
\hline \multicolumn{7}{|l|}{ Age (years) } \\
\hline$\leq 50$ & 16 & 2 & 5 & 5 & 4 & $>0.05$ \\
\hline$>50$ & 65 & 19 & 14 & 23 & 9 & \\
\hline \multicolumn{7}{|c|}{ Diameter of tumor $(\mathrm{cm})$} \\
\hline$\leq 2$ & 38 & 3 & 10 & 16 & 19 & $<0.01$ \\
\hline$>2$ and $\leq 5$ & 32 & 11 & 7 & 10 & 4 & \\
\hline$>5$ & 11 & 7 & 2 & 1 & 1 & \\
\hline \multicolumn{7}{|c|}{ Armpit lymph node metastasis } \\
\hline- & 50 & 6 & 11 & 22 & 11 & $<0.01$ \\
\hline+ & 31 & 15 & 8 & 6 & 2 & \\
\hline \multicolumn{7}{|c|}{ Histological grade } \\
\hline I & 11 & 0 & 0 & 5 & 6 & $<0.01$ \\
\hline II & 30 & 3 & 4 & 16 & 7 & \\
\hline II-III & 17 & 7 & 5 & 5 & 0 & \\
\hline III & 23 & 11 & 10 & 2 & 0 & \\
\hline
\end{tabular}

PDX-1, pancreatic-duodenal homeobox 1.

Table III. P53, Ki-67 and PDX-1 protein expression in breast cancer biopsies.

\begin{tabular}{|c|c|c|c|c|c|c|c|}
\hline \multirow{2}{*}{$\begin{array}{l}\text { Variable/ } \\
\text { grade }\end{array}$} & \multirow{2}{*}{$\begin{array}{c}\text { Total } \\
\text { specimen } \\
\text { number }(\mathrm{n})\end{array}$} & \multicolumn{4}{|c|}{ PDX-1 } & \multirow[b]{2}{*}{ P-value } & \multirow[b]{2}{*}{$\mathrm{R}$} \\
\hline & & - & + & ++ & +++ & & \\
\hline \multicolumn{8}{|l|}{ P53 } \\
\hline- & 48 & 9 & 10 & 17 & 12 & $<0.05$ & -0.321 \\
\hline+ & 17 & 5 & 4 & 7 & 1 & & \\
\hline++ & 10 & 4 & 4 & 2 & 0 & & \\
\hline+++ & 6 & 3 & 1 & 2 & 0 & & \\
\hline \multicolumn{8}{|l|}{ Ki-67 } \\
\hline- & 12 & 1 & 2 & 6 & 3 & $<0.01$ & -0.388 \\
\hline+ & 40 & 7 & 9 & 16 & 8 & & \\
\hline++ & 22 & 9 & 7 & 5 & 2 & & \\
\hline+++ & 6 & 4 & 1 & 1 & 0 & & \\
\hline
\end{tabular}

PDX-1, pancreatic-duodenal homeobox 1. 


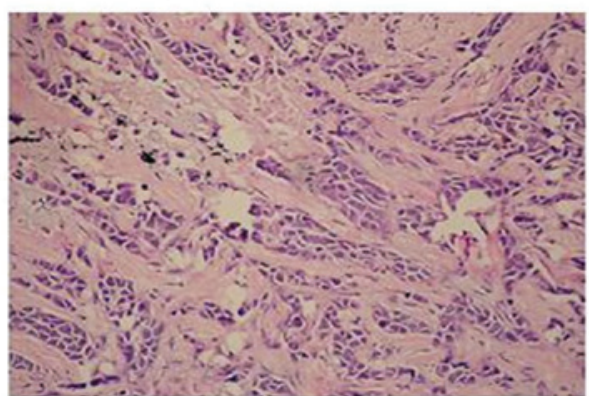

Breast cancer specimen (HE, SP $\times 200)$

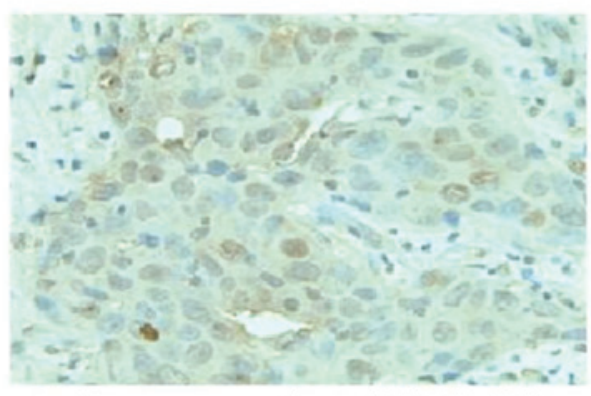

Breast cancer specimen (IHC, $\mathrm{SP} \times 400)$

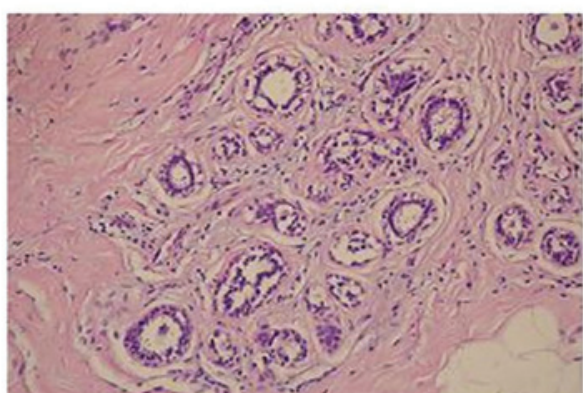

Adjacent normal breast tissue ( $\mathrm{HE}, \mathrm{SP} \times 200)$

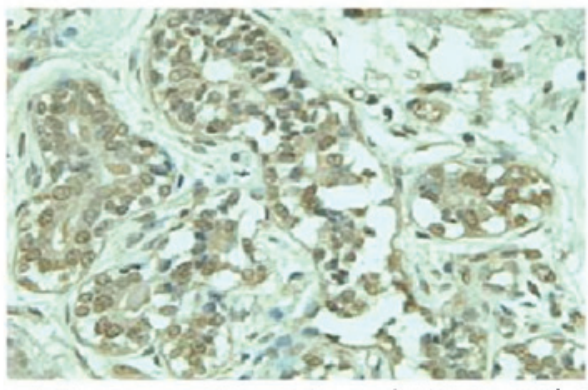

Adjacent normal breast tissue (IHC, $\mathrm{SP} \times 400$ )

Figure 1. HE and IHC detection of PDX-1 in breast cancer specimens and normal breast tissues. PDX-1 expression was strong in adjacent normal breast tissues. With cytoplasmic expression, PDX-1 was present in the nucleus as well, particularly on the nuclear membrane. HE, hematoxylin and eosin; IHC, immunohistochemical; PDX-1, pancreatic-duodenal homeobox 1.

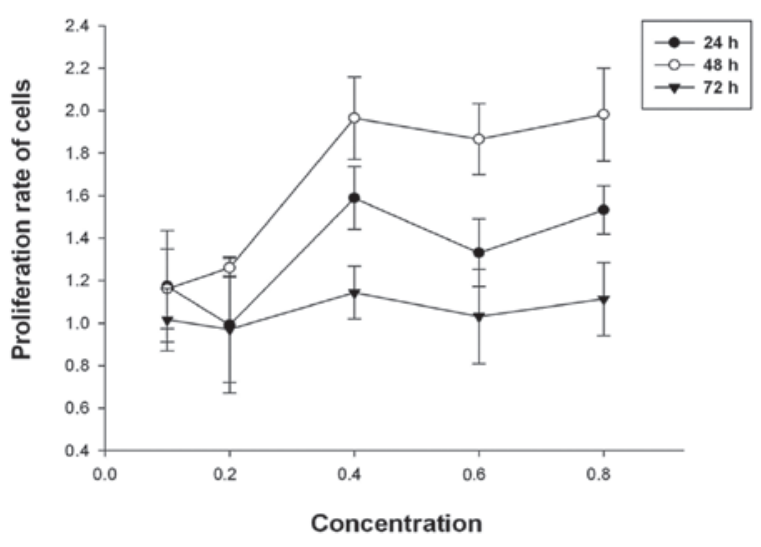

Figure 2. PDX-1 inhibits the proliferation of breast cancer cells. Transient transfection of PDX-1 ASO for $48 \mathrm{~h}$ at the concentration of $0.4 \mu \mathrm{mol} / 1$ enhanced the proliferation of breast cancer cells, compared with lipofect controls, $\mathrm{P}<0.05, \mathrm{n}=3$. PDX-1, pancreatic-duodenal homeobox 1; ASO, antisense oligonucleotide.

the positive rate of PDX-1 in breast cancer tissues was $71.6 \%$ (58/81), whereas that in non-cancerous tissues was $100 \%$ (20/20). The expression level of PDX-1 protein in breast cancer specimens was lower when compared with adjacent normal breast tissues $(\mathrm{P}<0.05$; Table I). Furthermore, PDX-1 expression was mainly cytoplasmic in breast cancer specimens. With cytoplasmic expression, PDX-1 was also present in the nucleus of adjacent normal breast tissues. Notably, it was particularly expressed on the nuclear membrane (Fig. 1). Statistical analysis revealed that the abnormal expression of PDX-1 in breast cancer negatively correlated with pathological grade $(\mathrm{P}<0.01)$, as well as the protein expression of P53 $(\mathrm{P}<0.05)$ and $\mathrm{Ki}-67$ $(\mathrm{P}<0.01$; Tables II and III).
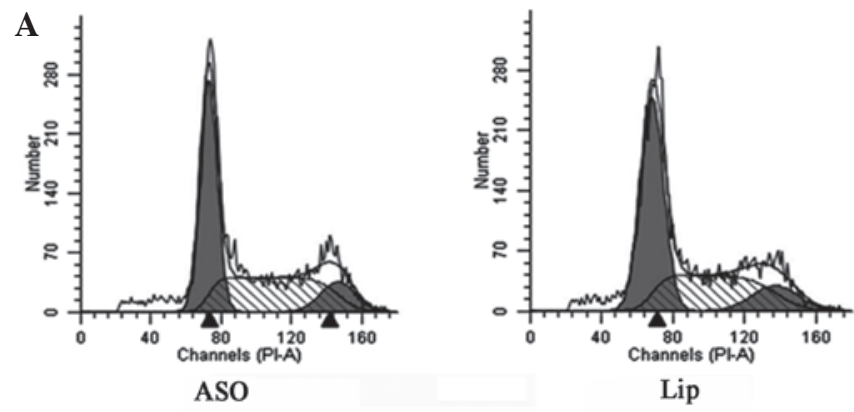

B

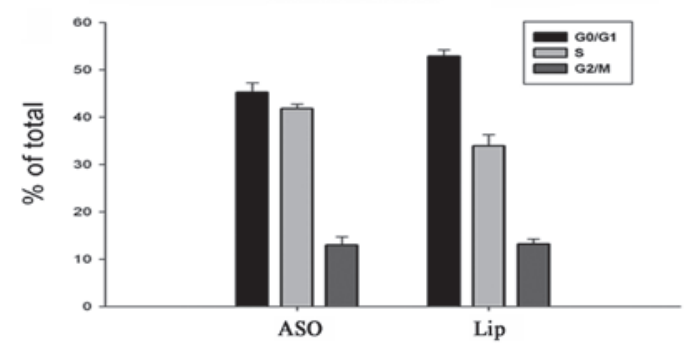

Figure 3. Effects of PDX-1 on the cell cycle in MCF-7 breast cancer cells (A) Representative analysis of the cell cycles following transfection with PDX-1 ASO. (B) Quantification of the number of cells at each stage of the cell cycle in PDX-1 ASO-transfected cells (ASO) and lipofect control (Lip). PDX-1, pancreatic-duodenal homeobox 1; ASO, antisense oligonucleotide.

PDX-1 ASO significantly inhibits PDX-1 expression in MCF-7 breast cancer cells. To investigate the role of PDX-1 in breast cancer, ASO-mediated knockdown was used. First, the transfection of PDX-1 ASO was optimized and validated. In comparison to lipofect controls, significantly lower expression of PDX-1 was observed $24 \mathrm{~h}$ after transfection of MCF-7 cells with PDX-1-specific ASO at concentrations of 0.1, 0.2, 0.4, 0.6 and $0.8 \mu \mathrm{mol} / 1$. Of these doses, the strongest inhibitory effect 
A

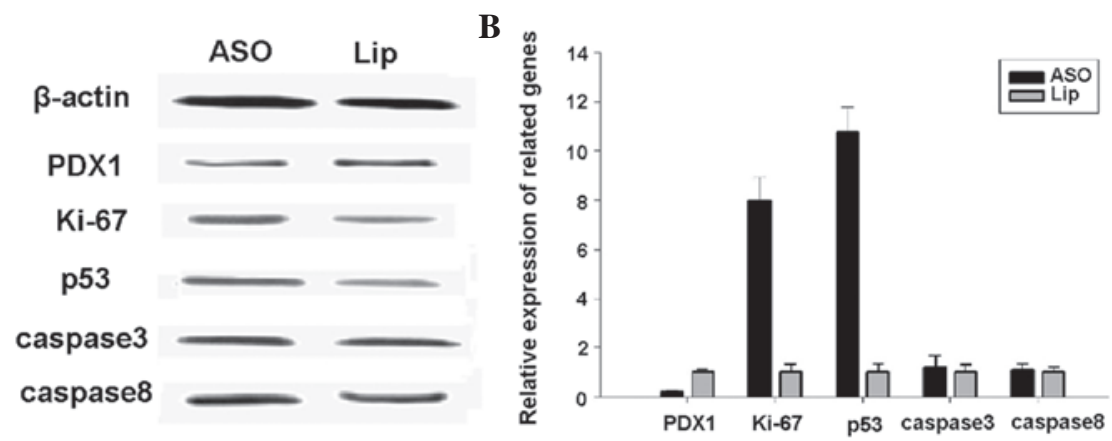

Figure 4. Effects of PDX-1 ASO on expression of PDX-1, Ki-67, P53, Caspase 3 and Caspase 8. (A) A representative western blot analyzing various proteins as indicated in lysates from PDX-1 ASO transfected MCF-7 cells (ASO) and control (Lip). (B) Quantification of three independent experiments PDX-1, pancreatic-duodenal homeobox 1; ASO, antisense oligonucleotide.

on PDX-1 mRNA expression was obtained with a PDX-1 ASO concentration of $0.4 \mu \mathrm{mol} / 1$ (data not shown). The relative PDX-1 gene expression compared to the lipofect-only control measured by real-time PCR was $0.495 \pm 0.023,0.321 \pm 0.023$, $0.045 \pm 0.006,0.096 \pm 0.011,0.147 \pm 0.006$ for $0.1,0.2,0.4$, $0.6,0.8 \mu \mathrm{mol} / 1$ of PDX-1 ASO, respectively $(\mathrm{P}<0.05, \mathrm{n}=3)$. In accordance with RT-PCR results, similar suppression of PDX-1 protein by PDX-1 ASO was observed with a transfection concentration of $0.4 \mu \mathrm{mol} / 1$ (data not shown).

PDX-1 inhibits growth of MCF-7 breast cancer cells. To characterize the impact of PDX-1 on the MCF-7 breast cancer cell line, we used PDX-1 ASO to downregulate PDX-1 and evaluate the growth rate of MCF-7 breast cancer cells at 24, 48 and $72 \mathrm{~h}$ after transfection using the CCK- 8 assay. The relative proliferation of PDX-1 ASO-transfected cells was higher than those of controls $(\mathrm{P}<0.05)$. PDX-1 ASO most effectively promoted the growth of MCF-7 breast cancer cells at $48 \mathrm{~h}$ and at a concentration of $0.4 \mu \mathrm{mol} / 1$ (Fig. 2). These data suggest that PDX-1 negatively regulates MCF-7 breast cancer cell proliferation.

PDX-1 modulates the cell cycle in MCF-7 breast cancer cells. To test whether PDX-1 modulates the cell cycle of breast cancer cells, PDX-1 ASO $(0.4 \mu \mathrm{mol} / \mathrm{l})$ was used to transfect MCF-7 breast cancer cells and $48 \mathrm{~h}$ later cell cycle analysis was performed using flow cytometry. In comparison to lipofectonly treated groups and negative controls, the percentage of G0/G1 phase cells $(48.25 \pm 0.87 \%$; $\mathrm{P}<0.05)$ markedly decreased in PDX-1 ASO transfected cells, while the proportion of $S$ phase cells $(39.63 \pm 0.90 \% ; \mathrm{P}<0.05)$ increased. The percentage of $\mathrm{G} 2 / \mathrm{M}$ phase cells was not significantly altered between the treatment and control groups. Furthermore, the cellular proliferation index rose to $55.59 \pm 0.77 \%(\mathrm{P}<0.05)$. This suggests that PDX-1 initiates G0/G1 phase arrest. The downregulation of PDX-1 expression with ASO led to an increased number of Sphase cells and higher proliferation index (Fig. 3A and B).

PDX-1 significantly downregulates P53 and Ki-67 expression in MCF-7 breast cancer cells. Real-time PCR was employed to test the expression levels of several genes involved in the cell cycle and apoptosis pathways. Remarkably, the expression of P53 and Ki-67 increased markedly following downregulation of PDX-1 when compared with controls, (the relative expression was $9.36 \pm 2.116$ for $\mathrm{P} 53$ and $7.985 \pm 0.939$ for $\mathrm{Ki}-67 ; \mathrm{P}<0.05$ $\mathrm{n}=3$ ). No significant alteration of Caspase 3 or Caspase 8 expression was observed (relative expression was $1.213 \pm 0.489$ for Caspase 3 and $1.113 \pm 0.247$ for Caspase 8, $P>0.05, n=3$; Fig. 4A). Consistent with RT-PCR findings, protein translation of P53 and Ki-67 was elevated following ASO-mediated knockdown of PDX-1, while the expression of Caspase 3 and 8 was not significantly altered. Together, these data demonstrate that silencing of PDX-1 with ASO induces the upregulation of P53 and Ki-67 (Fig. 4B).

\section{Discussion}

Breast cancer is the leading cause of cancer-related mortality in women worldwide (18). To date, tumorigenesis and progression of breast cancer remains unclear. However, genetic abnormalities have been shown to play a pivotal role.

Notably, we identified that the expression of PDX-1 in breast cancer tissues was not as strong as that in adjacent normal tissues. Moreover, PDX-1 was expressed in the nucleus of adjacent normal breast tissues, particularly on the nuclear membrane, suggesting that PDX-1 may function at the nuclear membrane. As to the fate of PDX-1 in breast cancer, Spearman's analysis indicated that PDX-1 negatively correlates with pathological stage and histological grade, as well as expression of P53 and $\mathrm{Ki}-67$. Thus, underexpression of PDX-1 may represent a lower pathological stage, histological grade and improved prognosis. Therefore, these data suggest that PDX-1 may be valuable as a new prognostic biomarker for breast cancer.

In further studies, ASO-mediated downregulation of PDX-1 expression facilitated the proliferation of MCF-7 breast cancer cells. In cell cycle assays, it was observed that lowering the level of PDX-1 markedly increased the number of S phase cells, suggesting that PDX-1 is a negative regulator of cell proliferation. Given the relatively low expression of PDX-1 in breast cancer tissues and its inhibitory impact on the growth of MCF-7 breast cancer cells, it is reasonable to assert that PDX-1 serves as a tumor suppressor gene in the tumorigenesis and maintenance of breast cancer. Similar to this study, Ma et al revealed that PDX-1 functions as a tumor suppressor in gastric cancer (16). 
In summary, the current study shows that PDX-1 is ubiquitously expressed in non-cancerous breast tissues and that its expression profile is different to that of breast cancer tissues, where loss of its expression might act as an early biomarker for breast cancer. We propose that PDX-1 functions as a tumor suppressor in breast cancer as evidenced by its ability to inhibit cancer cell proliferation and disrupt the cell cycle. In the future, PDX-1 may serve as a potential diagnostic and therapeutic target for breast cancer treatment.

\section{References}

1. Schwitzgebel VM, Mamin A, Brun T, et al: Agenesis of human pancreas due to decreased half-life of insulin promoter factor 1 . J Clin Endocrinol Metab 88: 4398-4406, 2003.

2. Fukuda A, Kawaguchi Y, Furuyama K, et al: Loss of the major duodenal papilla results in brown pigment biliary stone formation in pdx1 null mice. Gastroenterology 130: 855-867, 2006.

3. Gannon M, Tweedie Ables E, Crawford L, et al: Pdx-1 function is specifically required in embryonic $\beta$ cells to generate appropriate numbers of endocrine cell types and maintain glucose homeostasis. Dev Biol 314: 406-417, 2008.

4. Ashizawa S, Brunicardi FC and Wang XP: PDX-1 and the pancreas. Pancreas 28: 109-120, 2004.

5. Offield MF, Jetton TL, Labosky PA, et al: PDX-1 is required for pancreatic outgrowth and differentiation of the rostral duodenum. Development 122: 983-995, 1996.

6. Liu T, Gou SM, Wang CY, et al: Pancreas duodenal homeobox-1 expression and significance in pancreatic cancer. World J Gastroenterol 13: 2615-2618, 2007.

7. Jonmarker S, Glaessgen A, Culp WD, et al: Expression of PDX-1 in prostate cancer, prostatic intraepithelial neoplasia and benign prostatic tissue. APMIS 116: 491-498, 2008.
8. Leys CM, Nomura S, Rudzinski E, et al: Expression of PDX-1 in human gastric metaplasia and gastric adenocacinoma. Hum Pathol 37: 1162-1168, 2006

9. Sakai H, Eishi Y, Li XL, et al: PDX1 homeobox protein expression in pseudopyloric glands and gastric carcinomas. Gut 53: 323-330, 2004

10. Ballian N, Liu SH and Brunicardi FC: Transcription factor PDX-1 in human colorectal adenocarcinoma: A potential tumor maker. World J Gastroenterol 14: 5823-5826, 2008.

11. Wang XP, Li ZJ, Magnusson J, et al: Tissue microarray of pancreatic duodenal homeo-box-1 in human cancers. World J Surg 29: 334-338, 2005.

12. Tirone TA, Wang XP, Templeton NS, et al: Cell-specific cytotoxicity of human pancreatic adenocarcinoma cells using rat insulin promoter thymidine kinase directed gene therapy. WJ Surg 28: 826-833, 2004.

13. Liu SH, Patel S, Gingras MC, et al: PDX-1: demonstration of oncogenic properties in pancreatic cancer. Cancer 117: 723-733, 2011.

14. Koizumi M, Doi R, Toyoda E, et al: Increased PDX-1 expression is associated with outcome in patients with pancreatic cancer Surgery 134: 260-266, 2003.

15. Quint K, Stintzing S, Alinger B, et al: The expression pattern of PDX-1, SHH, Patched and Gli-1 is associated with pathological and clinical features in human pancreatic cancer. Pancreatology 9: 116-126, 2009.

16. Ma J, Chen M, Wang J, et al: Pancreatic duodenal homeobox-1 (PDX1) functions as a tumor suppressor in gastric cancer. Carcinogenesis 29: 1327-1333, 2008.

17. Livak KJ and Schmittgen TD: Analysis of relative gene expression data using real-time quantitative PCR and the $2-\Delta \Delta \mathrm{Ct}$ method. Methods 25: 402-408, 2001.

18. World Health Organization. Cancer fact sheet No. 297. Geneva, 2006. 